\title{
The gluon Sivers asymmetry measurements at COMPASS
}

\author{
Adam Szabelski on behalf of the COMPASS collaboration* \\ University of Trieste and INFN \\ E-mail: adam.szabelski@cern. ch
}

\begin{abstract}
The Sivers function describes the correlation between the transverse spin of a nucleon and the transverse motion of its partons. As such, a nonzero Sivers effect for gluons could be a signature of their nonzero orbital angular momentum inside the nucleon. COMPASS has collected data of semi-inclusive deep inelastic scattering by impinging $160 \mathrm{GeV} / c$ muons on transversely polarised proton and deuteron targets. The gluon Sivers asymmetry is extracted from a high- $p_{T}$ hadron pair sample with the use of monte carlo simulations and the a neural network approach. The results of a similar analysis for a Collins-like asymmetry for gluons will also be given.
\end{abstract}

XXV International Workshop on Deep-Inelastic Scattering and Related Subjects 3-7 April 2017

University of Birmingham, $U K$

\footnotetext{
* Speaker.
} 


\section{Introduction}

Sivers asymmetry is a semi-inclusive measure of the modulation amplitude of the Sivers angle distribution of the hadrons produced in deep inelastic scattering (DIS) of a lepton off a transversely polarised nucleon target. When it is non-zero the parton distribution in the transversely polarised nucleon is not left-right symmetric with respect to the plane defined by the directions of nucleon spin and momentum [1]. The left-right asymmetry was first observed in pions produced in $p^{\uparrow} p \rightarrow$ $\pi X$ [2]. This asymmetry is connected to the parton orbital angular momentum (OAM) which was first noticed by Sivers [1]

Later experimental results came from HERMES Collaboration [3] and then by the COMPASS Collaboration [4] where the Sivers asymmetry was observed by scattering leptons off transversely polarised proton targets. Since then the experimental effort to measure the Sivers effect for quarks was continued by HERMES, COMPASS and JLab.

Much less is known about the Sivers asymmetry for gluons and it is interesting due to its connection with gluon OAM. It is theoretically constrained by the so-called Burkardt sum rule [5]. The first phenomenological result based on PHENIX data using the transverse single spin asymmetry $A_{N}\left(p^{\uparrow} p \rightarrow \pi^{0} X\right)$ was derived [6] giving constrains on the gluon Sivers function.

For DIS in this analysis free single-photon exchange processes are taken into account. The leading process (LP) $\gamma^{*} q \rightarrow q$, QCD Compton (QCDC) $\gamma^{*} q \rightarrow q g$ and photon-gluon fusion (PGF) $\gamma^{*} q \rightarrow q \bar{q}$. From these processes only the latter gives information about the gluon. Selection of a pure PGF sample is impossible. In this analysis we use the method of weights applied already in $\Delta G / G$ extraction [8]. More details on the present analysis can be found in [7].

Another analysis based on $J / \Psi$ production is also covered in this text in Sect. 2. The other sections are dedicated to the gluon Sivers from SIDIS analysis.

\section{The gluon Sivers asymmetry measurement via $J / \Psi$ production at COMPASS}

The Sivers asymmetry in $J / \Psi$ production in scattering of muons off transversely polarised protons $\mu^{+}+p^{\uparrow} \rightarrow \mu^{\prime}+J / \Psi+X$ is measured in two $z$-bins in the COMPASS 2010 data. Events with three muons in the final state are selected. The invariant mass distributions of the muon pairs of opposite signs are shown in Figure 1 separately for the two $z$-bins together with the boundaries of the signal band and two side-bands, which are used to evaluate the asymmetry of the background. The signal to background and signal to total ratios were calculated to be $N_{s i g} / N_{b g}=4.3, N_{\text {sig }} / N_{\text {tot }}=$ 0.8 for the first bin in $z$ and $N_{\text {sig }} / N_{b g}=5.2, N_{\text {sig }} / N_{\text {tot }}=0.8$ for the second. The Sivers asymmetry is the amplitude of the modulation $\sin \left(\phi_{p_{T}}-\phi_{S}\right)$, where $p_{T}$ is the transverse (with respect to the virtual photon) momentum of the reconstructed $J / \Psi$. It is assumed that the background asymmetry is the same as the side-band asymmetry and is subtracted. The results is shown in Fig. 2 (right). The obtained asymmetries, $A_{\text {Siv }}^{p}=-0.05 \pm 0.33$ for $z \in[0.3 ; 0.95]$ and $A_{S i v}^{p}=-0.28 \pm 0.18$ for $z \in[0.95 ; 1.05]$, show the tendency to the negative sign but the statistical error is large and it is difficult to draw any resolute conclusion. The recent theoretical predictions [9] give a negative value consistent with our measurement. 

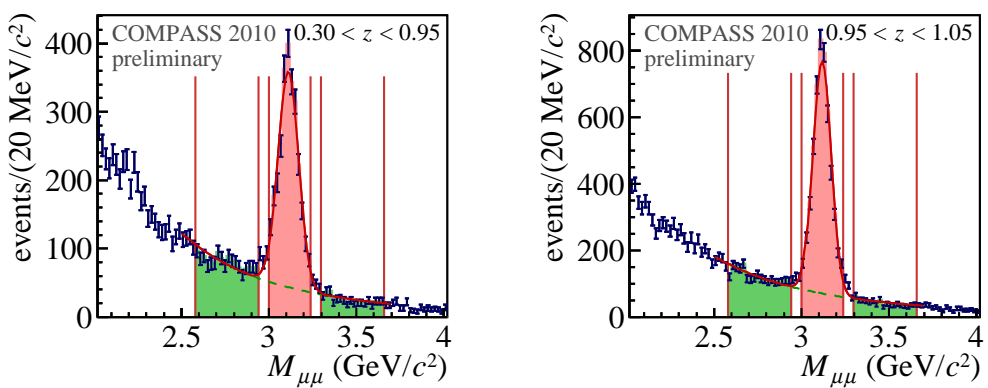

Figure 1: Dimuon invariant mass in the two z-intervals. Left: inclusive events , right: exclusive events. The boundaries of the side-bands and the signal band are denoted by vertical red lines. The red fit to the data is the normal distribution plus background in the form $A N\left(M_{\mu \mu}, \mu, \sigma\right)+B M_{\mu \mu}^{C}$. The dotted green line is the background estimation $B M_{\mu \mu}^{C}$.
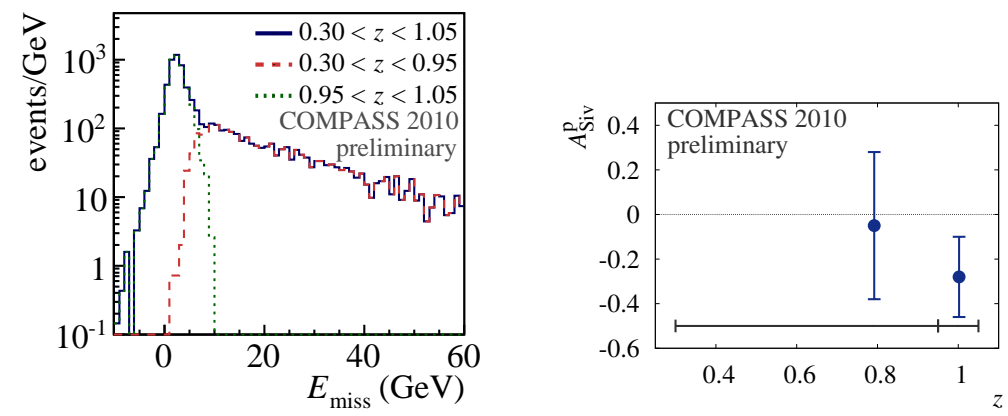

Figure 2: Missing energy spectrum (left) and the final Sivers asymmetry in the two $z$ bins (right).

\section{COMPASS set-up and data selection}

The COMPASS experiment is a fixed target experiment placed at CERN in the north area. It uses a polarised muon beam from SPS. In this analysis two data sets where used: data collected in 2003-4 with the transversely polarised ${ }^{6} \mathrm{LiD}$ target (deuteron data) and data collected in 2010 with transversely polarised $\mathrm{NH}_{3}$ target (proton data). The ${ }^{6} \mathrm{LiD}$ target consisted of two oppositely polarised cells while the ammonia target of three cells with the central cell polarised antiparallel to the outer cells. The cell polarisation was reversed once per week which enables cancellation of the acceptance effects in the analysis. The basic features of the COMPASS spectrometer, as described in Ref. [10], are the same for 2003-4 and 2010 data taking.

The main goal of the data selection is to access the gluon azimuthal angle $\phi_{g}$. It was checked using monte carlo (MC) that the strongest correlation is between $\phi_{g}$ and the azimuthal angle of the vector sum $P$ of the leading and next-to-leading hadron momenta, $\phi_{P}$ and this correlation is even stronger when demanding high $p_{T}$ of the two hadrons. Keeping in mind that the high- $p_{T}$ selection reduces the statistics the requirement is chosen to $p_{T 1}>0.7 \mathrm{GeV} / c$ and $p_{T 2}>0.4 \mathrm{GeV} / c$. Moreover, the fractional energies of the two hadrons must fulfil the conditions $z_{i}>0.1 \quad(\mathrm{i}=1,2)$ and $z_{1}+z_{2}<0.9$. The photon virtuality is chosen to be $Q^{2}>1(\mathrm{GeV} / \mathrm{c})^{2}$ and the mass of the hadronic final state, $W>5 \mathrm{GeV} / c^{2}$. The Bjorken- $x$ variable covers the range $0.003<x_{B j}<0.7$. 
The fractional energy of the virtual photon is limited by $0.1<y<0.9$.

\section{Asymmetry extraction using the method of weights}

In order to extract the gluon asymmetry from PGF events a sample of events with two hadrons in the final state is used. The Sivers Asymmetry can then be written as

$$
A_{T}^{2 h}\left(\vec{x}, \phi_{\text {Siv }}\right)=\frac{\Delta \sigma\left(\vec{x}, \phi_{\text {Siv }}\right)}{\sigma(\vec{x})},
$$

here $\vec{x}=\left(x_{B j}, Q^{2}, p_{T 1}, p_{T 2}, z_{1}, z_{2}\right), \Delta \sigma \equiv d^{7} \sigma \uparrow-d^{7} \sigma \downarrow$ and $\sigma \equiv d^{7} \sigma \uparrow+d^{7} \sigma \downarrow$. The number of events in a $\phi_{S i v}$ bin is given by $N\left(\vec{x}, \phi_{S i v}\right)=\alpha\left(\vec{x}, \phi_{S i v}\right)\left(1+f(\vec{x}) P_{T} A^{S i v}(\vec{x}) \sin \phi_{S i v}\right)$. Here $f$ is the dilution factor, $P_{T}$ is the target polarisation and $\alpha$ is an acceptance-dependent factor. The Sivers asymmetry $A_{T}^{2 h}\left(\vec{x}, \phi_{S i v}\right)$ is factorised into the amplitude $A^{\operatorname{Siv}}(\vec{x})$ and the modulation $\sin \phi_{S i v}$.

The model with only PGF, QCDC and LP processes is successful in describing the unpolarised data. The LP is dominating the cross-section; the other two can be enhanced, however, by selecting hadrons with high $p_{T}$. Introducing the processes fractions $R_{j}(j=P G F, Q C D C, L P)$ the amplitude of the Sivers asymmetry can be expressed in terms of the amplitudes of the three contributing processes:

$$
A^{S i v}=R_{P G F} A_{P G F}^{S i v}+R_{Q C D C} A_{Q C D C}^{S i v}+R_{L P} A_{L P}^{S i v}
$$

The weighted method used in this analysis was already applied to extract the gluon polarisation from the longitudinal double-spin asymmetry in the SIDIS measurement of single hadron production [8]. While the method may appear somewhat complex, the basic idea behind is rather simple. Equation (4.2) contains three unknowns, i.e. the asymmetries $A_{j}^{S i v}$, so that for a solution at least three equations of the type of Eq. (4.2) are needed. These three equations are constructed using the weighting procedure described in this section, which in addition allows us to achieve a nearly optimal statistical accuracy.

Both the deuteron runs (two target cells) and for the proton run (three target cells) four target configurations can be introduced. Decomposing the Sivers asymmetry into the asymmetries of the contributing processes (Eq. (4.2)) and introducing the Sivers modulation $\beta_{j}^{t}\left(\phi_{S i v}\right)=R_{j} f P_{T}^{t} \sin \phi_{S i v}$ specific for the process $j$ the number of events is given by

$$
\begin{aligned}
N^{t}\left(\vec{x}, \phi_{S i v}\right)=\alpha^{t}\left(\vec{x}, \phi_{S i v}\right) & \left(1+\beta_{P G F}^{t}\left(\phi_{S i v}\right) A_{P G F}^{S i v}(\vec{x})\right. \\
& \left.+\beta_{Q C D C}^{t}\left(\phi_{S i v}\right) A_{Q C D C}^{S i v}(\vec{x})+\beta_{L P}^{t}\left(\phi_{S i v}\right) A_{L P}^{S i v}(\vec{x})\right)
\end{aligned}
$$

where $t=1,2,3,4$ denotes the target configuration. For each process a statistical weighting factor is introduced which is chosen to be $\omega_{j} \equiv \beta_{j} / P_{T}$. Each of the four equations (4.3) is weighted three times with $\omega_{j}$ depending on the process $j \in(\mathrm{PGF}, \mathrm{QCDC}, \mathrm{LP})$ and integrated over $\phi_{S i v}$ and $\vec{x}$ giving twelve observed quantities $q_{j}^{t}$ :

$$
\begin{aligned}
q_{j}^{t} & =\int d \vec{x} d \phi_{S i v} \omega_{j}\left(\phi_{S i v}\right) N^{t}\left(\vec{x}, \phi_{S i v}\right) \approx \sum_{i=0}^{N^{t}} \omega_{i}^{j} \\
& =\tilde{\alpha}_{j}^{t}\left(1+\left\{\beta_{P G F}^{t}\right\} \omega_{j}\left\{A_{P G F}^{S i v}\right\}_{\beta_{P G F}^{t} \omega_{j}}+\left\{\beta_{Q C D C}^{t}\right\} \omega_{j}\left\{A_{Q C D C}^{S i v}\right\}_{\beta_{Q C D C}^{t} \omega_{j}}+\left\{\beta_{L P}^{t}\right\} \omega_{j}\left\{A_{L P}^{S i v}\right\}_{\beta_{L P}^{t} \omega_{j}}\right),
\end{aligned}
$$


where $\tilde{\alpha}^{t}=\int d \vec{x} d \phi_{S i v} \alpha^{t} \omega$ is the weighted acceptance-dependent factor and the quantities $\left\{\beta_{i}^{t}\right\} \omega_{j}$ and $\left\{A_{i}^{\text {Siv }}\right\}_{\beta_{i}^{t} \omega_{j}}$ are weighted averages:

$$
\{\beta\}_{\omega}=\frac{\int \alpha \beta \omega d \vec{x} d \phi_{\mathrm{Siv}}}{\int \alpha \omega d \vec{x} d \phi_{\mathrm{Siv}}},\{A\}_{\beta \omega}=\frac{\int A \alpha \beta \omega d \vec{x} d \phi_{\mathrm{Siv}}}{\int \alpha \beta \omega d \vec{x} d \phi_{\mathrm{Siv}}} .
$$

The weighted acceptance-dependent factors cancel assuming their ratio is the same before and after the polarisation reversal, $\left(\tilde{\alpha}_{j}^{1} \tilde{\alpha}_{j}^{4}\right) /\left(\tilde{\alpha}_{j}^{2} \tilde{\alpha}_{j}^{3}\right)=1$.

\section{Monte Carlo optimisation and Neural Network training}

The analysis which has been performed is very similar to the one described in [8]. In this analysis the package NetMaker [11] is used. The package provides NN training with custom input, output and target vector. The NN has been trained with a Monte Carlo sample with process identification. As an input vector six kinematic variables have been chosen, $x_{B j}, Q^{2}, p_{T 1}, p_{T 2}, p_{L 1}, p_{L 2}$. The latter two are the longitudinal components of the hadron momenta. The trained neural network is applied to the data by taking the vector of the aforementioned six variables. Hence, the simulated distribution of these variables need to be in agreement with their distributions in the data samples and it was checked to be the case. The main goal of of NN parameterisation is an estimation of $R_{j}$. In the present analysis one has to estimate simultaneously the fractions of the three processes. The NN returns three $R_{j}$ values for each process adding up to one. Studies on MC data show that the average $R_{j}$ values from the NN and the true fractions $R_{j}$ from the MC process ID are consistent.

\section{Results}

The method presented in Sect. 4 with the use of trained NNs has been applied to the two data sets. The gluon contribution to the Sivers asymmetry is shown in Fig. 3 together with the contribution of the two other two hard processes. The result of the analysis of the deuteron data is $A_{P G F}^{S i v, d}=-0.14 \pm 0.15$ (stat.) \pm 0.06 (syst.) measured at $\left\langle x_{g}\right\rangle=0.13$. It is consistent with the results for proton data, $A_{P G F}^{S i v, p}=-0.26 \pm 0.09$ (stat.) \pm 0.08 (syst.) obtained at $\left\langle x_{g}\right\rangle=0.15$ within one standard deviation of the total uncertainty (obtained as linear combination of the statistical and systematic uncertainty). This compatibility is expected as presumably the transverse motion of gluons is the same in deuteron and proton.

While the gluon contribution to the Sivers asymmetry is found to be consistent with zero for the COMPASS deuteron data its value for the proton data is below zero by more than two standard deviations. This result is interesting comparing to the recent analysis of the PHENIX data, [6], which show compatibility with zero of the gluon Sivers effect for protons.

The positive value obtained for for high- $p_{T}$ sample of the COMPASS proton data for the asymmetry of the leading process, can be compared with the COMPASS results for the SIDIS single hadron measurement [12]. There, the asymmetry for negative hadrons is found to be about zero and that for positive hadrons above zero, so that for the two-hadron final state a positive value may be expected. 

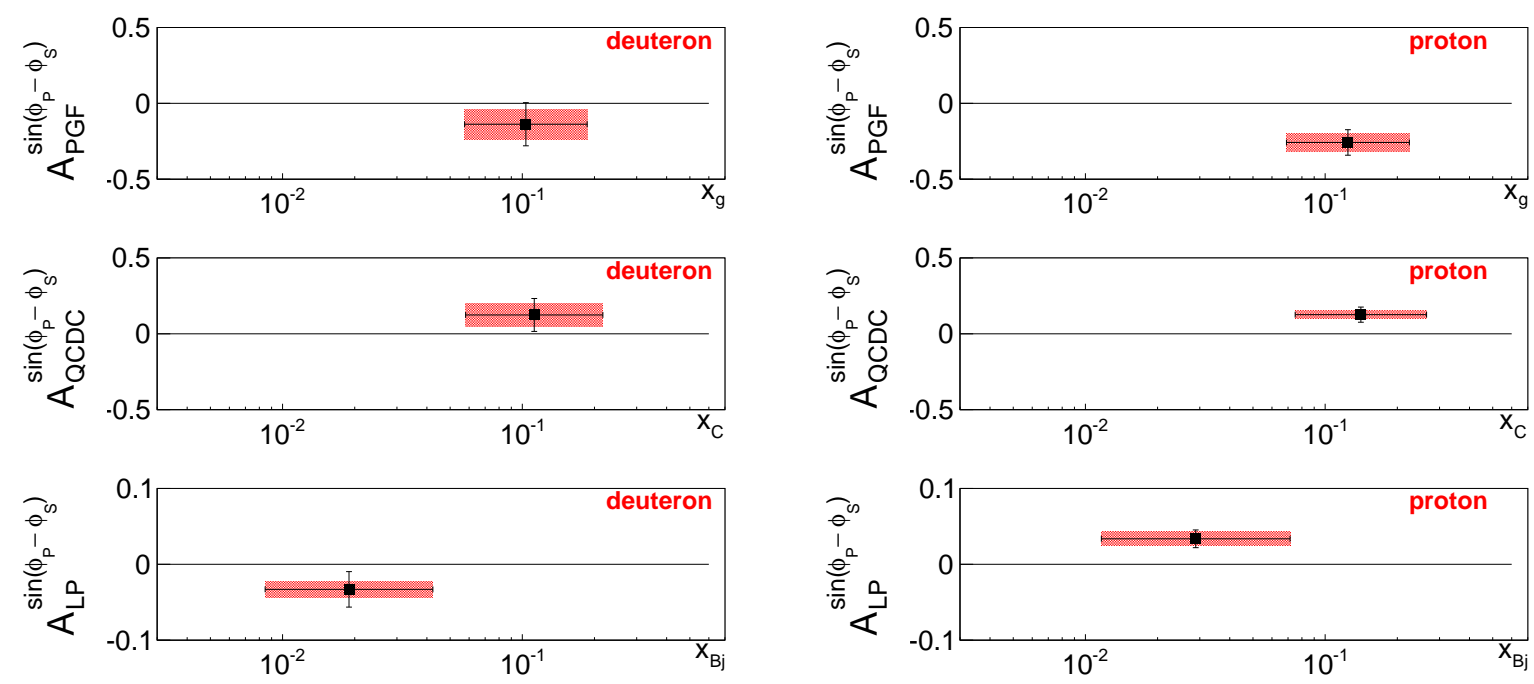

Figure 3: Sivers two-hadron asymmetry extracted for Photon-Gluon fusion (PGF), QCD Compton (QCDC) and Leading Process (LP) from the COMPASS high- $p_{T}$ deuteron (left) and proton (right) data. The $x$ range is the RMS of the logarithmic distribution of $x$ in the MC simulation. The red bands indicate the systematic uncertainties. Note the different ordinate scale used in the third row of panels.
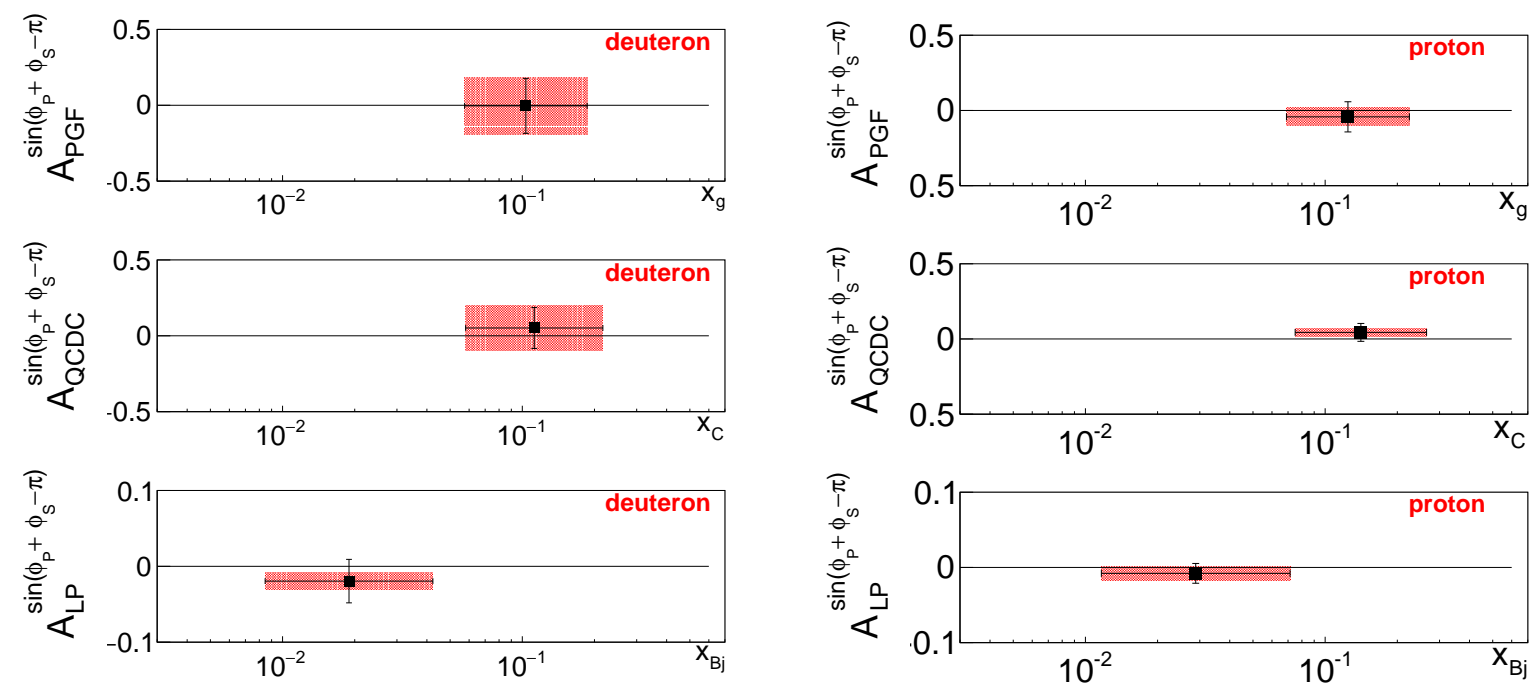

Figure 4: Collins-like two-hadron asymmetry extracted for Photon-Gluon fusion (PGF), QCD Compton (QCDC) and Leading Process (LP) from the COMPASS two-hadron high- $p_{T}$ deuteron (left) and proton (right) data. The $x$ range is the RMS of the logarithmic distribution of $x$ in the MC simulation. The red bands indicate the systematic uncertainties.

The procedure was repeated for a Collins-like modulation with the different azimuthal angle definition, $\phi_{C o l}=\phi_{P}+\phi_{S}-\pi$. The results, shown in Fig. 4, are compatible with zero. 


\section{References}

[1] D. W. Sivers, Phys. Rev. D41, 83 (1990).

[2] J. Antille et al., Phys. Lett. B94, 523 (1980).

[3] HERMES Collaboration, A. Airapetian et al., Phys. Rev. Lett. 94, 012002 (2005), hep-ex/0408013.

[4] COMPASS Collaboration, M. G. Alekseev et al., Phys. Lett. B692, 240 (2010), hep-ex/1005.5609.

[5] M. Burkardt, Phys. Rev. D69, 091501 (2004), hep-ph/0402014.

[6] U. D’ Alesio, F. Murgia, and C. Pisano, JHEP 09, 119 (2015), hep-ph/1506.03078.

[7] COMPASS Collaboration C. Adolph et al.,hep-ex/1701.02453.

[8] C. Adolph et al. [COMPASS Collaboration], Eur. Phys. J. C 77 (2017) no.4, 209, hep-ex/1512.05053.

[9] A. Mukherjee and S. Rajesh, arXiv:1609.05596 [hep-ph]

[10] COMPASS Collaboration, P. Abbon et al., Nucl. Instrum. Meth. A577, 455 (2007), hep-ex/0703049.

[11] R. Sulej, Netmaker, http://www.ire.pw.edu.pl/ rsulej/NetMaker/.

[12] COMPASS Collaboration, C. Adolph et al., Phys. Lett. B717, 383 (2012), hep-ex/1205.5122. 